\title{
Eye movements and brain electric potentials during reading
}

\author{
Reinhold Kliegl • Michael Dambacher • \\ Olaf Dimigen - Arthur M. Jacobs · Werner Sommer
}

\begin{abstract}
The development of theories and computational models of reading requires an understanding of processing constraints, in particular of timelines related to word recognition and oculomotor control. Timelines of word recognition are usually determined with event-related potentials (ERPs) recorded under conditions of serial visual presentation (SVP) of words; timelines of oculomotor control are derived from parameters of eye movements (EMs) during natural reading. We describe two strategies to integrate these approaches. One is to collect ERPs and EMs in separate SVP and natural reading experiments for the same experimental material (but different subjects). The other strategy is to co-register EMs and ERPs during natural reading from the same subjects. Both strategies yield data that allow us to determine how lexical properties influence ERPs (e.g., the N400 component) and EMs (e.g., fixation durations) across neighboring words. We review our recent research on the effects of frequency and predictability of words on both EM and ERP measures with reference to current models of eye-movement control during reading. Results are in support of the proposition that lexical access is distributed across several fixations and across brain-electric potentials measured on neighboring words.
\end{abstract}

R. Kliegl (ه) - M. Dambacher - O. Dimigen Department of Psychology, University of Potsdam, Karl-Liebknecht-Str. 24-25, 14476 Potsdam, Germany e-mail: kliegl@uni-potsdam.de

M. Dambacher - A. M. Jacobs

Freie Universität Berlin, Berlin, Germany

O. Dimigen - W. Sommer

Humboldt Universität zu Berlin, Berlin, Germany

\section{Introduction}

Reading unfolds in time. Measurement of event-related potentials (ERPs) derived from the electroencephalogram (EEG) yield the highest resolution of time-related constraints in reading. Natural reading unfolds also in space that is across the lines of text. Measurement of eye movements (EMs) yield the precise information about "space-related" constraints associated with reading. Traditionally, ERPs and EMs have been obtained in separate experiments with rather different experimental designs. In our research we try to relate EMs and ERPs in the context of natural left-to-right reading. We describe two strategies to integrate time-related and space-related approaches. One is to collect ERPs and EMs in separate SVP and natural reading experiments for the same experimental material (but different subjects). The other strategy is to co-register EMs and ERPs during natural reading from the same subjects. Both strategies yield data that allow us to determine how properties of words in a sentence (e.g., the type frequency of words and their predictability from prior context) influence ERPs (e.g., the N400 component) and EMs (e.g., fixation durations) across neighboring words.

\section{Reading from an ERP-research perspective}

When skilled readers move their gaze across lines of printed text in order to make sense of letter sequences and spaces, it is very likely that for each word an elementary set of mental operations is repeated. These operations compute a form representation of the physical signal, match it with abstract representations stored in long-term memory, and select a (best) candidate for identification (Grainger \& Jacobs, 1996). This basic process, generally referred to as 
lexical access, has been one of the major issues in cognitive psychology in the past three decades (for review, see Carr \& Pollatsek, 1985; Jacobs \& Grainger, 1994).

Event-related potentials provide the highest resolution about the timing of language-related processes. Three ERP components are in the focus of current research: the leftanterior negativity (LAN), the P600, and the N400. LANs are associated with extra effort that needs to be dispensed when dealing with unexpected, incorrect, or difficult syntactic constructions (e.g., King \& Kutas, 1995); the P600 component appears when a reanalysis of the syntactic frame is called for (Hagoort \& Brown, 2000), that is, in the presence of morphosyntactic or syntactic violations. Of particular relevance for normal language processing is the N400 component, which appears to serve as an indicator of lexical preactivation (Kutas \& Federmeier, 2000, 2011) and lexical-semantic integration effort (e.g., Hagoort, Baggio, \& Willems, 2009).

There are two limitations associated with ERPs. First, ERP components are not process-pure measures. For example, the N400 component is not a purely linguistic component and is, for example, also elicited by arithmetic material (Niedeggen \& Rösler, 1999; Niedeggen, Rösler, \& Jost, 1999; Jost, Hennighausen, \& Rösler, 2004). From recent summaries of lesion, fMRI-, and ERP studies (Friederici \& Weissenborn, 2007; Hagoort \& van Berkum, 2007; Rösler, 2011), it appears that the N400 indicates regulation of the relative activation level of representations of the posterior parts of the brain, mostly declarative and procedural parts of semantic memory. In this context, the N400 is interpreted as a sign of additional activation in these posterior areas. The component is modulated if new, not yet expected representations of content and structure must be accessed. The N400 component appears to be relevant for both language-related and memory-related processing.

The second limitation relates to the generalization of ERP results. In ERP experiments subjects are usually instructed to hold fixation at the center of the screen where words of the sentence are presented at a fixed rate, usually in the range of 500-1000 ms per word [i.e., serial visual presentation (SVP) paradigm]. The procedure is used mainly for two reasons. First, SVP avoids the need for eye movements, which are known to contribute large electroocular artifacts to the EEG. Indeed, the effects triggered by an eye movement can be magnitudes larger than typical psycholinguistic effects (Picton et al. 2000). Second, the long interval between words as compared with the average fixation duration of around $200-250 \mathrm{~ms}$ in natural reading serves to prevent overlap of brainwaves triggered by successively presented words. The procedure may be perfectly acceptable if the goal is to understand word recognition in the context of sentences, but, obviously, the differences between SVP and natural reading may lead to processing differences. In contrast to natural reading there is no preview of upcoming words and, if oculomotor control interacts with lexical processing, there may be effects on timelines of word recognition due to the absence of saccades. ${ }^{1}$

\section{Reading from an eye-movement control perspective}

Obviously, research on eye-movement control during reading presupposes eye movements. For natural reading it has been established beyond doubt that fixation durations are highly valuable indicators of local processing difficulty. Whatever property or statistic makes a word more difficult (e.g., low frequency low predictability from prior sentence context) is very likely to increase fixation durations. By one account, there are over 50 known correlated properties of a word relevant for its visual recognition (Graf, Nagler, \& Jacobs, 2005). Potentially, all of them influence eyemovement parameters and quite a few of them have been established for many different languages (e.g., Rayner, 1998, 2009, for reviews). Despite their reliability and validity as indicators of psycholinguistic processing, fixations do not inform us about timelines associated with lexical or sublexical processes within their duration. This is their major limitation in comparison with ERPs. ${ }^{2}$

\section{Perceptual span and spatial attention}

The focus of EM control research has been on spatial constraints during reading. Two main lines of interrelated research can be distinguished: (a) delimiting the spatial zone of influence on fixation durations (the when? question) and (b) the dynamics of oculomotor control (saccade programs for selection of fixation locations) and shifts of spatial attention (the where? question). The spatial zone of influence is known as the perceptual span and was mapped out with gaze-contingent display change experiments. In such experiments, letters outside a predefined number of letters to the left or right of the current fixation location are masked (moving window paradigm; McConkie \& Rayner, 1975) or a critical target word is not revealed until the eyes cross an invisible boundary placed before the word (boundary paradigm; Rayner, 1975). Since masking letters about 15 characters to the right and 4 characters to the left of the point of fixation does not impede reading rate, this

\footnotetext{
1 The instruction to keep a constant point of fixation does not preclude fixational eye movements. They contribute to ERPs and may generate interpretative problems under some conditions (Dimigen, Valsecchi, Sommer, \& Kliegl, 2009)

2 The potential of fixational movements (i.e., microsaccades, drift, and tremor during a fixation) has not been tapped systematically.
} 
zone has been called the perceptual span. Determining which type of information (i.e., visual, sublexical, lexical, syntactic, semantic, pragmatic) becomes available at which moment during the fixation and from which locations relative to the current fixation position is critical for deciding between competing theoretical proposals about eye-movement control in reading that attempt to model the dynamics of oculomotor control and spatial attention shifts. Two major classes of models are currently distinguished: models proposing that saccade targeting is guided by processing gradients, which assume that several words may be processed simultaneously (e.g., Engbert \& Kliegl, 2011; Engbert, Nuthmann, Richter, \& Kliegl, 2005; Inhoff, Radach, Starr, \& Greenberg, 2000; Kennedy, 2000; Reilly \& Radach, 2006) and models proposing serial attention shifts from one word to the next (e.g., Engbert \& Kliegl, 2001; Morrison, 1984; Reichle, Pollatsek, Fisher, \& Rayner, 1998; Reichle, Warren, \& McConnell, 2009). These views of lexical processing during reading have also been implemented in computational models, but neither the current state of these computational models nor the current state of experimental/empirical results affords clear-cut predictions to decide between them. They do play a very useful role in formulating coherent theoretical positions (Kliegl \& Engbert, 2011) as well as in inspiring multivariate analyses of eye movements and new experiments. Therefore, we briefly describe two of these models, $E-Z$ Reader (Reichle et al., 1998, 2009) and SWIFT (Ėngbert et al., 2005), and then introduce a series of theoretical issues from their perspectives as a blueprint for the coordination of EM and ERP research (for a comprehensive review of other models, see Reichle, Rayner, \& Pollatsek, 2003).

\section{E-Z Reader}

A very simple notion linking fixations and lexical processing is embodied in the immediacy-of-processing assumption: fixation durations reflect the time needed to comprehend a currently fixated word $N^{3}$ (Just \& Carpenter, 1980). This assumption is wrong and no current model adheres to it in a strict fashion. For example, in E-Z Reader (Reichle et al., 1998, 2009) a saccadic program towards word $N+1$ is initiated after an early familiarity check (stage L1) of word $N$ is completed. During saccade programming, word $N$ is further processed and usually lexically accessed before the saccade is executed. The completion of lexical access (L2) of word $N$ in $E$-Z Reader is considered as "a point when processing of a word is

\footnotetext{
${ }^{3}$ In the following, word $N$ always denotes the location of a critical fixation and word $N-1$ and word $N+1$ are its direct left and right neighbors, respectively.
}

complete enough so that the processing system can shift attention to the next word so that lexical processing of that word can begin with no 'crosstalk' from the processing of the prior word." (Pollatsek, Reichle, \& Rayner, 2006, p. 11). Thus, according to E-Z Reader, only one word is processed at a time and does not interact with recognition of consecutive words. Nevertheless, the word being processed is not necessarily the fixated word. Of course, at this point in time, the attention shift during a reading fixation is purely hypothetical, but there may be behavioral (e.g., in properties of fixational eye movements such as microsaccades or drift) or neural correlates of such discrete shifts of sequential attention (e.g., linked to a timeline associated with an ERP component).

\section{SWIFT}

SWIFT (autonomous Saccade generation With Inhibition by Foveal Targets; Engbert et al., 2005) postulates only a loose relation between oculomotor control and language processing and distributed processing during reading. Distributed processing means that several words are processed in parallel at a rate decreasing with the eccentricity of the word relative to the point of fixation. ${ }^{4}$ Lexical processing is implemented as two successive diffusion processes with the first one randomly walking towards a maximum of lexical activity (lexical preprocessing) linked to word difficulty (i.e., the inverse of word frequency) and the second one randomly walking back towards zero (lexical completion). Processing rates towards and back from the peak differ by a preprocessing factor. In general, saccade programs are triggered at random time intervals; thus, unlike in $E-Z$ Reader they are not directly linked to lexical processes (i.e., the start of the second diffusion process). The height of lexical activity on the fixated word, however, inhibits (i.e., delays) the next saccade program. This mechanism generates a dependency of single and first fixation durations on word difficulty.

\section{Limitations}

The models recover a range of benchmark effects about fixation durations and probabilities as a function of word frequency. Nevertheless, both of them still work with a very simple interface to the text. For example, they assume that readers know the location of words on a line, that is, their beginnings and ends, demarcated by spaces. In addition to the spatial layout of words in the sentence, type frequency (usually operationalized as the log frequency per

\footnotetext{
4 This notion of "distributed processing" should not be confused with the distinction between "distributed" (i.e., connectionist) and localist models of word recognition (Jacobs \& Grainger, 1994).
} 
million printed words) and predictability of words (i.e., the conditional probability that a word is guessed given the earlier words of the sentence) are assumed to translate into processing difficulty. There is a rough distinction between two lexical processing stages, but not much is said about theoretical issues that define current research in word recognition. In the following section, we review some of the issues that sketch the zone of proximal development for future models of eye-movement control during reading in this respect. The joint consideration of ERP and EM measures is required to become more precise about timelines of word recognition during reading.

\section{Theoretical issues}

\section{Lexical accèss and oculomotor control}

The timing of mental events that take place during a fixation has been the object of many theoretical proposals and controversial debates in the literature ever since Gough's (1972) paper "one second of reading", in which he claimed a left-to-right processing of the letters of the fixated word (at a rate of $10 \mathrm{~ms} / \mathrm{letter}$ ). Gaze-contingent movingwindow and boundary experiments aimed to determine the relative and absolute times at which stimulus information becomes available (McConkie, Underwood, Zola, \& Wolverton, 1985)). Sereno, Rayner, \& Posner, 1998 focused on lexical access as the central process during reading around which all other events are situated. While lexical access plays a major role in models of isolated word recognition (MROM, Grainger \& Jacobs, 1996; DRC, Coltheart, Rastle, Perry, Langdon, \& Ziegler, (2001)) and models of eye movement control during reading (SWIFT, Engbert et al., 2005; E-Z Reader, Reichle et al., 2009; Glenmore, Reilly \& Radach, 2006), there is still no general agreement among researchers with regard to fundamental questions, namely (a) how it works, (b) when during a fixation it starts and stops, and (c) to what extent it contributes to eye movement control. The timeline of lexical access and oculomotor control will remain a major research focus for the years to come.

Lag and successor effects

Based on a large corpus of reading eye-movements, Kliegl, Nuthmann, \& Engbert, 2006 presented evidence that single-fixation durations in first-pass reading carry reliable information not only about the fixated word $N$ but also about word $N-1$ and word $N+1$. They refer to effects of word $N-1$ and word $N+1$ on fixation durations on word $N$ as lag and successor (word) effects, respectively. The reasoning about influences of neighboring words can be summarized in five principles (Kliegl et al., 2006): (1) Processing in the perceptual span occurs in parallel, although with rates decreasing with eccentricity. (2) Cognitive load dynamically modulates the extent of the perceptual span, which implies a distinction between the actual and the potential perceptual span (e.g., Henderson \& Ferreira, 1990; Rayner \& Pollatsek, 1987). (3) Word recognition and semantic access continue to influence fixation durations after the eyes have moved, setting up a cognitive processing lag. (4) Memory retrieval of a predictable parafoveal word $N+1$ may lead to processing this word during the fixation on word $n$ and increases the associated fixation duration on word $n$. (5) Longer fixation durations in the center of words are due to a higher probability of quick restarts of saccade programs at the edges of words as a consequence of misplaced fixations (Nuthmann, Engbert, \& Kliegl, 2005). Similar proposals have been presented earlier (e.g., Inhoff et al., 2000; Kennedy, Pynte, \& Ducrot, 2002; Kolers, 1976).

\section{Competing explanations of lag effects}

One strong lag effect is that fixation durations on word $N$ are longer when the preceding stimulus (i.e., word $N-1$ ) is of low frequency (Kliegl et al., 2006; Rayner \& Duffy, 1986). E-Z Reader and SWIFT differ in their explanation of this effect. E-Z Reader explains the effect primarily (not exclusively) in terms of reduced parafoveal preview rather than in terms of ongoing processing: after lexical access of word $N-1$ attention immediately shifts to word $N$, while the saccade to word $N$ usually occurs with some delay. Thus, a difficult word $N-1$ grants less time to process word $N$ in the parafovea. Longer fixation durations on word $N$ are therefore due to less preview of this word during the fixation on word $N-1$. In contrast, when SWIFT encounters a difficult word $N-1$, the lexical processing system is able to inhibit the autonomous saccade generator, which increases fixation duration on word $N-1$. However, because the cortical word recognition processes are much slower than the fast brainstem saccade generator, this inhibition process could be delayed (Engbert et al., 2005) and potentially arises only during the next fixation. Consequently, inspection durations on word $N$ following a fixation on a difficult word $N-1$ are prolonged, because processing of word $N-1$ was incomplete and needs to be completed during subsequent fixations (i.e., spillover, Rayner \& Duffy, 1986). Thus, while both models can account for this lag effect, EEG signals that represent a reliable timeline of lexical processing during reading fixations may constrain current computational models or, more likely, their successors enough to render one of the two explanations (i.e., reduced preview time or spillover) as less plausible than the other. 


\section{Competing explanations of successor effects}

Of theoretical concern is also conflicting evidence about preprocessing effects between corpus-analytic and experimental evidence. Preprocessing effects are due to parafoveal preview obtained of word $N+1$, and manifest either during the fixation on word $N$ or when finally fixating on word $N+1$. Parafoveal preview effects can be demonstrated with boundary experiments. When word $N+1$, the word directly to the right of the boundary, is masked prior to fixation, fixations on this word are longer in comparison with a condition in which word $N+1$ is visible during the fixation on word $N$. The difference in fixation durations between the two conditions is called preview benefit. Although less consistent, there is also evidence for so-called parafoveal-on-foveal effects reflecting the influence of properties of the parafoveal word $N+1$ on fixation durations on the currently fixated word $N$ (for a review on parafoveal preprocessing see Rayner, White, Kambe, Miller, \& Liversedge, 2003). In contrast to boundary experiments, there is highly reliable evidence for parafoveal-on-foveal effects of word $N+1$ frequency and predictability in corpus analyses (e.g., Kennedy \& Pynte, 2005; Kliegl, 2007; Kliegl et al., 2006). The differences between boundary experiments and corpus analyses are probably due to (a) the larger range of frequency and predictability values of words included in corpus analysis, (b) lower statistical power of experimental manipulations, and (c) selection of different words for the analysis, or a combination of them (see Rayner, Pollatsek, Drieghe, Slattery, \& Reichle, 2007; Kliegl, 2007, for a discussion). Preview benefit and parafoveal-on-foveal effects hold the potential to test differences between accounts in terms of sequential attentions shifts or processing gradients. According to sequential attention shifts as implemented in $E-Z$ Reader only sublexical, but not lexical information is extracted from the parafovea, whereas accounts in terms of processing gradients as implemented in SWIFT do not impose such restrictions. Evidence for or against lexical preprocessing, however, does not immediately falsify these models. For example, although E-Z Reader makes the strong assumption that words are processed serially, it also allows for full processing of a word $N$ from word $N-1$ if the intended saccade undershot the intended word $N$ due to oculomotor error (i.e., an account in terms of mislocated fixations). In SWIFT lexical access of a parafoveal word is a function of the lengths, processing difficulties, and associated activations of words in the perceptual span. Thus, there usually are reasons why complete parafoveal lexical access may not occur. Again, EEG signals that demonstrate a reliable lexical preview benefit or parafoveal-on-foveal effect will contribute to the resolution of empirical inconsistencies, and facilitate the further development of computational models.

\section{Relating two observable time series of reading: eye movements and EEG}

Surprisingly, despite earlier proposals to relate ERP and EM research (Sereno \& Rayner, 2003; Sereno et al., 1998), this perspective has gained some momentum only in recent years. Here, we review results from our research (1) from studies that have collected ERPs and EMs during reading the same sentences, but with different groups of subjects (Dambacher, Göllner, Nuthmann, Jacobs, \& Kliegl, 2006; Dambacher \& Kliegl, 2007), and (2) from an experiment during which ERPs and EMs were recorded simultaneously in a single group of subjects (Dimigen, Sommer, Hohlfeld, Jacobs, \& Klieg1, 2011). Such co-registration research encounters not only quite a few technical and substantive problems, but also opportunities for a deeper understanding of the relation between oculomotor effects and brain signals (Dimigen, Sommer, Hohlfeld, Jacobs, \& Kliegl, 2011). In all experiments subjects read the Potsdam Sentence Corpus (PSC) that comprises frequency and predictability norms for each of its 1138 words (Kliegl, Grabner, Rolfs, \& Engbert, 2004).

\section{Graded effects of predictability on $\mathbf{N 4 0 0}$ and fixation durations during reading: separate traditions}

The role of the N400 component under conditions of mismatch has been and continues to be examined in the context of language-related processing. For example, if one hears the sentence "The opposite of black is white," there is only a very weak negativity associated with "white" because this is the expected color given the prior sentence context. The negativity, however, is clearly visible if "yellow" is presented instead of "white", because yellow is the "wrong" color for this sentence. Nevertheless, "yellow" still is color and consequently the negativity is less pronounced for this word in comparison with "nice" because in this case the mismatch extends to the category (see Fig. 1; after Roehm, Bornkessel-Schlesewsky, Rösler, \& Schlesewsky, 2007).

Such graded effects of predictability are of considerable interest for research on eye-movement control during reading where similar effects have been reported. Figure 2 (right panel) shows first-pass single-fixation durations (SFDs) during reading of isolated sentences related to the odds for correctly predicting the fixated word given the prior sentence context (Kliegl et al., 2006). SFDs are defined as events that are preceded by a saccade from a 
Fig. 1 Grand average ERPs for antonyms and related and unrelated conditions in Experiment 3 (onset at the vertical bar). Negativity is plotted upward. From Roehm et al. (2007; part of Fig. 2; Copyright (2007), with permission from MIT Press)
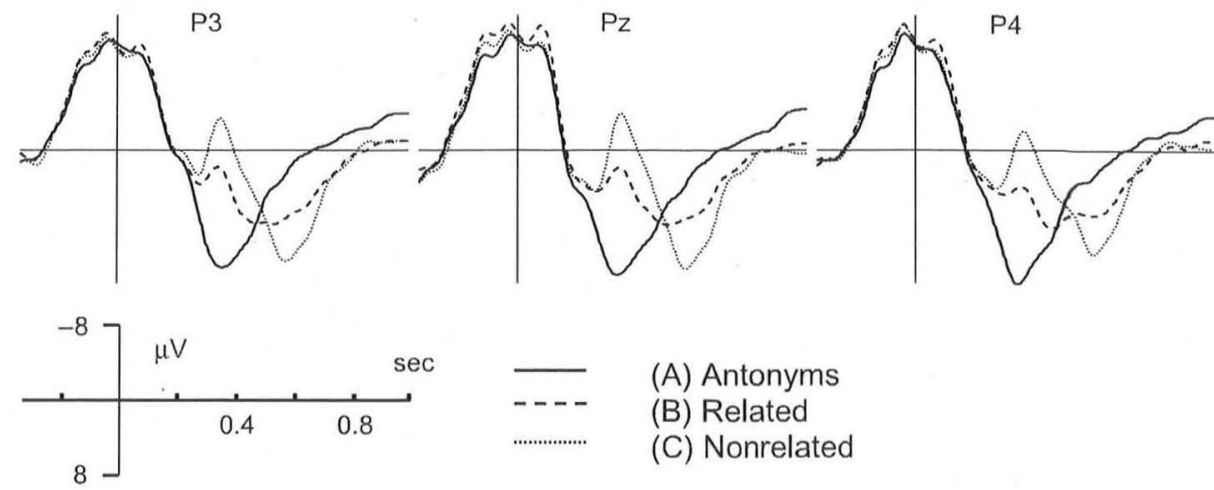

Fig. 2 Frequency and predictability effects on singlefixation duration during normal reading. From Kliegl et al. (2006; part of Fig. 3; Copyright (2006), with permission from American Psychological Association)

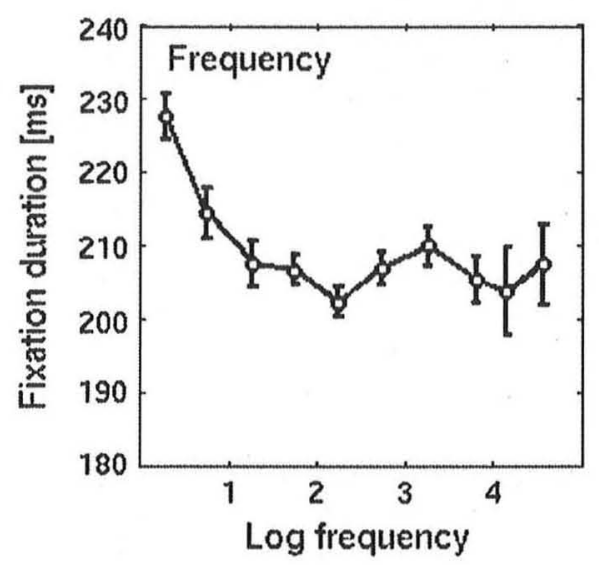

previous word and are followed by a saccade to a subsequent word before the eye had ever fixated or skipped the word before. The odds ratios for each word were collected in a separate study with a cloze procedure, involving almost 300 subjects, yielding 83 predictions for each word (Kliegl et al., 2004). Figure 2 (left panel) also displays the relation of SFDs to the logarithm of the printed type frequency of the fixated word. Overall, SFDs are shorter if words are of high predictability or high frequency. Are the graded effects of predictability on the N400 component of ERP research identical with the covariate effects of predictability on SFDs? The answer is not immediately evident because, as reviewed above, reading is studied under two very different situations. We have carried out experiments and multivariate analyses that attempt to delineate similarities and differences.

\section{Relating ERPs and SFDs using words (not subjects) as unit of observation}

In a first step we collected ERPs with a standard SVP procedure for the sentences that yielded the predictability and frequency effects on SFDs shown in Fig. 2. The study included a comparatively large sample of 48 subjects (Dambacher et al., 2006). Each word was presented for $250 \mathrm{~ms}$; the interval between stimulus onsets was $700 \mathrm{~ms}$. As shown in Fig. 3, for 343 open-class words (i.e., nouns, verbs, adjectives) the sentences yielded the expected graded effects of frequency and predictability on the N400 amplitude between 300 to $500 \mathrm{~ms}$ (average-referenced ERPs shown for channel $\mathrm{Pz}$ ).

The graded pattern of means of Fig. 3 provides face validity, but we can relate the critical results of the two experiments much more directly to each other by switching from the usual subject-based analysis to a word-based analysis (Dambacher \& Kliegl, 2007). Averaging across subjects within the two experiments we obtained SFDs and N400 amplitudes for each of the 343 open-class words and tested frequency and predictability effects for these data. Figure 4 displays the rather remarkable agreement of their mean profiles. We want to highlight the indication of a nonlinear profile for the frequency effects and a close to linear profile for predictability effects. Although not statistically significant in this analysis, the differential pattern is highly reliable for large corpora of EMs. Specifically, the transient increase in SFD for medium-to-high frequency values has been established as significant in nine different 
Fig. 3 Frequency and from Dambacher et al. (2006) predictability effects on $\mathrm{N} 400$ during SVP reading. Data are
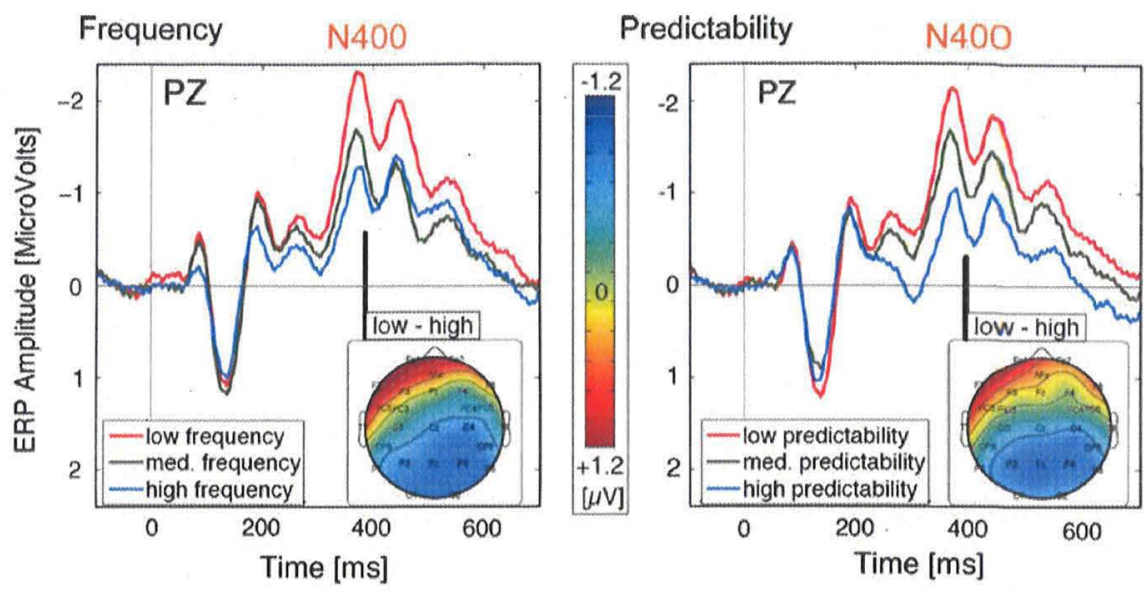

Fig. 4 Immediate relations: word $n$ effects on FDn and N400n. Mean fixation durations (FDn) and N400 amplitudes (N400n) of word $n$ as function of frequency (left panel) and predictability (right panel) of word $n$. Data points were calculated on the basis of quantiles for frequency and predictability. Error bars reflect 99\% confidence intervals. Eye movement data are from Klieg et al. (2006) and EEG data are from Dambacher et al. (2006). Figure from Dambacher and Kliegl (2007; Fig. 2; Copyright (2007), with permission from Elsevier)

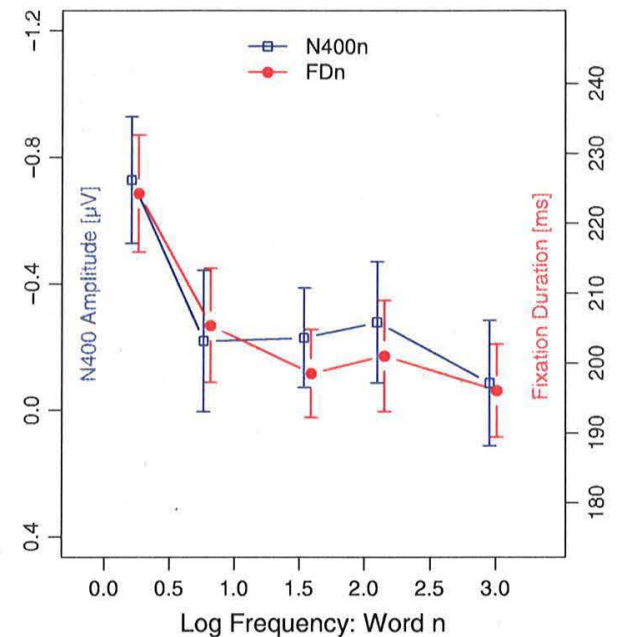

data sets (Klieg1, 2007). Such agreement is by no means trivial given the large differences between the two settings. In particular, given average fixation durations of around $200 \mathrm{~ms}$, the N400 component from 300 to $500 \mathrm{~ms}$ occurs at a time when the eyes have typically already moved on to the next word. Thus, neural activity related to processing difficulty of word $N$ (or word $N-1$ ) temporally overlaps with the fixation on word $N+1$ (or word $N$ ).

The similarity of effect profiles together with the diverging timelines calls for a multivariate analysis of the relations between these measures, taking also into account the correlation between ERPs and SFDs on neighboring words. This can be accomplished with a path analysis in which the hypothesized directions of influences between variables are specified in diagrams such as those shown in Fig. 5. Path analysis yields a confirmatory test statistic about the consistency between predictions of such models and a matrix of observed correlations. Correlations between successive measures of time series (such as successive fixation durations or successive ERPs) are specified as autoregressive effects (e.g., a parameter estimating the effect of regressing SFD on word $N$ on SFD on word $N-1$; Dambacher \& Kliegl, 2007). We distinguish between a baseline path model and a successor path model (see Fig. 5, panels a and b). As we modeled the correlations for words (not subjects) as observational units, our application represents a path analysis over items. The main result was that the observed correlation matrix is statistically consistent with the baseline model, assuming (a) that the N400 triggered by word $N-1$ is (negatively) correlated with SFD on the same word (i.e., red bidirectional arrow in Fig. 5a) and (b) that there is a negative correlation between the N400 triggered by word $N$ and SFD measured on word $N$ (i.e., blue bidirectional arrow in Fig. 5a). These patterns correspond to and re-establish the mean profiles of Fig. 4 in the context of the path analysis.

At the same time, the observed correlations were also consistent with the path-model predictions (a) that processing of word $N-1$ continues beyond the SFD on word $N-1$ and affects SFD on the next word $N$ and (b) that processing of word $N$ continues beyond the SFD on word $N$ and affects SFD on word $N+1$ (i.e., red and blue 
(a) Baseline Model

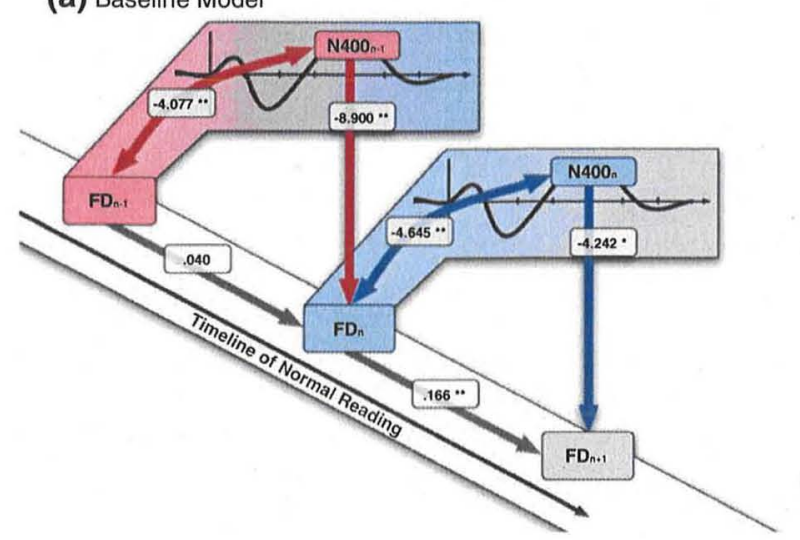

(b) Successor Effect Model

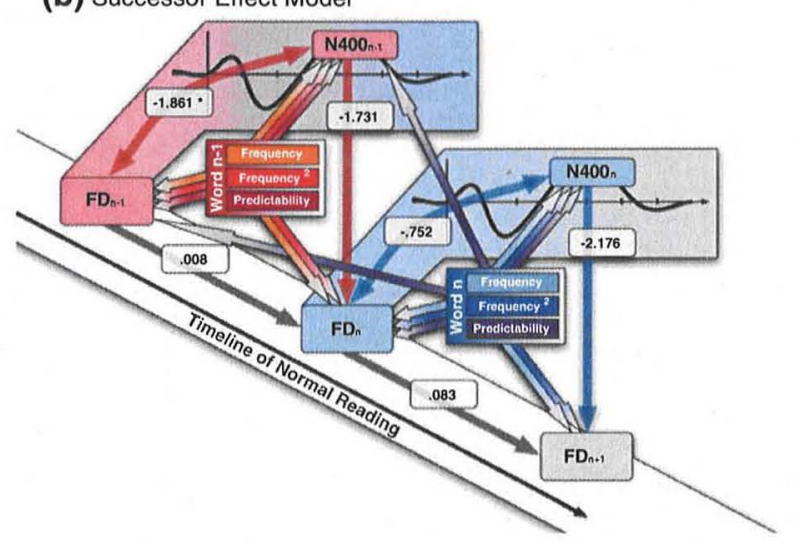

Fig. 5 Path-analytic models. Visualization of path analyses, together with path coefficients $\left({ }^{*} p<.05 ;{ }^{*} p<.01\right)$. a The baseline model, i.e., direct relations between $\mathrm{N} 400$ amplitudes and fixation durations (FD) across word triplets (word $N-1$, word $N$, word $N+1$ ). b The successor effect model comprising influence of word frequency and predictability in addition to paths in the baseline model. Directed paths from predictability of word $n$ back to $\mathrm{FD}_{N-1}$ and back to $\mathrm{N} 400_{N-1}$ were estimated with positive signs (i.e., as non-canonical effects). Figure from Dambacher and Kliegl (2007; Fig. 5; Copyright (2007), with permission from Elsevier)

unidirectional arrows in Fig. 5a, respectively). The justification for the specification of directed paths (i.e., postulates of a causal influences) from $\mathrm{N} 400$ (word $N-1$ ) on SFD (word $N$ ) and $\mathrm{N} 400$ (word $N$ ) on SFD (word $N+1$ ) derives from the joint consideration of SVP results for ERP assessment and SFD measurements. Due to the SOA of $700 \mathrm{~ms}$ in the ERP study N400 amplitudes are uniquely attributable to presentation and processing of the corresponding word. In other words, in the SVP-ERP experiment word $N$ was not presented at the time at which the N400 was triggered by word $N-1$ and word $N+1$ was not yet available during presentation of word $N$. Thus, the results are consistent with the prediction that $\mathrm{N} 400$ effects of the last word are one source of variance on subsequent fixation durations. The joint consideration of results from regular reading (Kliegl et al., 2006) and SVP reading (Dambacher et al., 2006) allows us to dissociate the influence of successive N400 components on successive reading fixations. Such analyses highlight how experimental control over the presentation afforded by the SVP procedure and natural reading complement each other in a productive way.

As reviewed above, there are two main explanations for lag effects between neighboring fixation durations: reduced preview benefit or spillover. Longer fixation durations on word $N$ due to reduced preview benefit after a difficult word $N-1$ is not a plausible interpretation for the pathanalytic N400-related effect from word $N-1$ on word $N$ because there is no preview in the SVP procedure. The path-analytic result is, however, compatible with the spillover account, that is, the notion that processing of difficult words $N-1$ continues during fixations on word $N$ (Rayner \& Duffy, 1986). Of course, these results do not rule out reduced preview benefit as a second source of lag effects during natural reading.

\section{Including effects of frequency and predictability in the path model}

There is, of course, the expectation that the pattern of correlations between SFDs and N400s of neighboring words depicted in Fig. 5a arises from the frequencies and predictabilities of words $N-1, N$, and $N+1$ and their respective influences on N400 and SFD. Dambacher and Kliegl (2007) arrived at the model depicted in Fig. 5b as a summary of the distributed processing during SFDs on word triplets with reference to associated N400 amplitudes. The model shown in Fig. $5 b$ provides an acceptable fit to the joint covariance matrix and recovers quite a large number of well-established facts relating both to processing of the fixated word (i.e., immediacy effects) and of the parafoveal word (i.e., successor effects). ${ }^{5}$

Briefly, as far as immediacy effects are concerned, frequency and predictability of the fixated word $N$ and the previous word $N-1$ relate negatively to SFDs and positively to N400s, as already expected from the mean profiles shown in Fig. 4. With these variables in the model, the direct paths between fixation durations are no longer significant (i.e., word $N$ ) or greatly reduced (i.e., word $N-1$ ). The remaining significance in the latter case is probably due to the lack of inclusion of word $N-2$ (or even larger

\footnotetext{
${ }^{5}$ Given the confirmatory logic associated with tests of goodness of fit of path models, it is reasonable to assume that there are alternative models with equally good or even better fit statistics. For details of model development and estimates of path coefficients we refer to the original article.
} 
lags). The reduction in the size of path coefficients can be seen from a comparison of Fig. 5a, b. In particular, the causal influence of N400 triggered by word $N-1$ and visible in the fixation duration on word $N$ as well as the analogous pattern involving word $N$ and word $N+1$ is much smaller after statistically controlling for these word properties. Thus, the results are consistent with the prediction that frequency and predictability of words are a common source for the correlation between fixation durations and N400 amplitudes.

Perhaps even more importantly, the path model also recovers a previously reported non-canonical positive effect of the predictability of word $N+1$ on the fixation duration on word $N$ (Kliegl et al., 2006). The effect is noncanonical because usually high predictability is assumed to shorten processing time, as indeed it is the case for the predictability of the fixated word $N$. This non-canonical effect of predictability of word $N+1$ is quite stable; it was found in nine separate data sets (Kliegl, 2007). Kliegl et al. proposed that processing of a word $N+1$ may occur during fixation on word $N$ and lead to longer fixation durations on this word if word $N+1$ can be predicted from prior sentence context, that is, if prior sentence context basically triggers automatic lexical access or retrieval of word $N+1$ from memory. The conditions under which such an effect occurs are not understood yet. In the context of natural reading, we do not know how much of the effect depends on perceptual support from parafoveal processing. It also depends on the lexical status of words $N$ and $N+1$ (i.e., it was significant if either word $N$ or word $N+1$ is a function word; Kliegl, 2007).

In the path analysis, we recovered the non-canonical predictability (i.e., positive) effect for the predictability of word $N$ on the fixation duration on word $N-1$ (which is also an effect of a next word on a fixated word). Importantly, there was also a non-canonical predictability effect of word $N$ on the N400 triggered by $N-1$ (only marginally significant). In this case, given that words $N-1$ and $N$ were not simultaneously present in the SVP paradigm, there was no perceptual support for this effect; its origin must come exclusively from the prediction generated by the prior sentence context, quite in agreement with the explanation of automatic lexical access or retrieval from memory. Indeed, the long ISI of $700 \mathrm{~ms}$ may "invite" the prediction of upcoming words to a greater degree than is the case during normal reading (Dambacher, 2010). The methodology of carrying out experiments under natural reading and under SVP conditions with the same reading material and using path analysis with words (rather than subjects) as units of observations to integrate and test patterns of effects derived from competing theoretical accounts holds much promise for reading research in general.

\section{Co-registration of EMs and EEG}

The approach described in the last section is one way to combine the two traditional strands of reading research. This first strand exploits the high temporal resolution of the EEG to examine the timeline of word recognition. However, due to methodological problems, such as EM artifacts in the EEG signal and overlap of ERPs to words in close temporal vicinity, it still relies on the rather artificial experimental setups with SVP. In the second strand of reading research, EMs are recorded during natural reading and the interplay of word recognition and oculomotor control is examined. This method permits more direct inferences about the timing of word recognition, which are not trivial if they can be combined, for example, with experimental manipulation of the availability of preview. By coregistrations of EMs and ERPs in natural reading situations, we take advantage of the strengths of both methods, exploring a new approach to examine natural reading.

In the study by Roehm et al. (2007), see Fig. 1), the authors presented the words of the sentences of their experiment one at a time in the SVP format, that is, in the center of the screen, obliviating any need for eye movements. In a later study, Kretzschmar, Bornkessel-Schlesewsky and Schlesewsky (2009) used the same sentence material, but had subjects read the sentences during normal left-to-right reading while they collected both EEG and EMs. To avoid eye movement artifacts, Kretzschmar et al. constrained the analysis to electrodes close to the vertex, which are not strongly influenced by horizontal saccades. ERPs time locked to the first fixation on the target word exhibited an N400 for low-predictability conditions. They also found longer first fixations for non-antonyms versus antonyms. Interestingly, they also reported an N400 modulation on the last fixation before the critical region (but no effect on the corresponding fixation duration to which this N400 was locked). Since this N400 modulation was significant in a time window when participants were typically already looking at the critical word, it is not clear whether it can be attributed to parafoveal processing. In any case, however, these results indicate that $\mathrm{N} 400$ effects arise early in natural reading.

We co-registered and analyzed EMs and ERPs for all words of the PSC in a natural, left-to-right fashion while 30 subjects read at their individual pace (Dimigen et al., 2011). A 32-channel EEG recording system was linked to a high-resolution infrared eye tracker. EEG segments were cut around fixations and each segment was then related to an extensive set of word properties retrieved from the PSC database. Furthermore, because the PSC was studied extensively with $\mathrm{EM}(N=222$ readers $)$ and ERPs using the RSVP technique ( $N=48$ readers, Dambacher et al., 2006) 
(a)
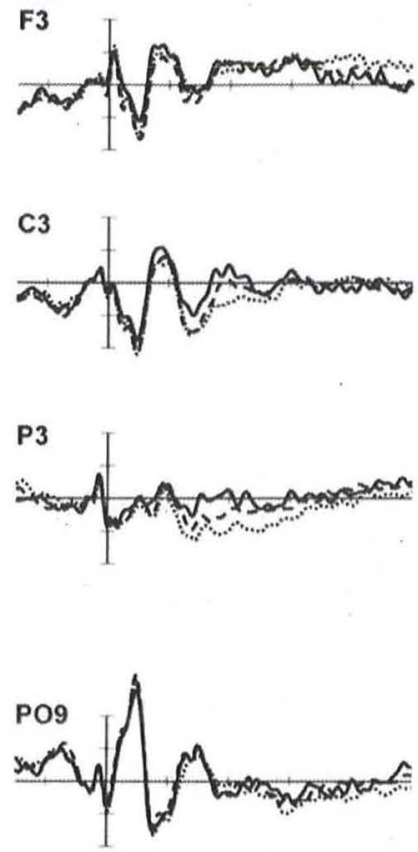

(b)

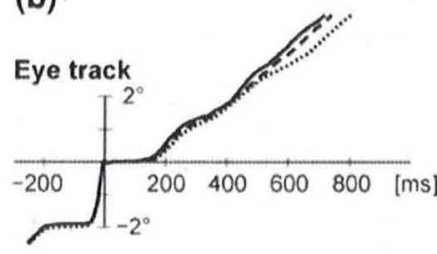

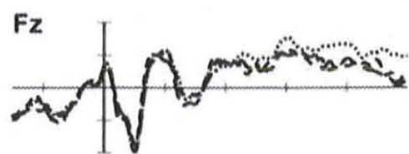
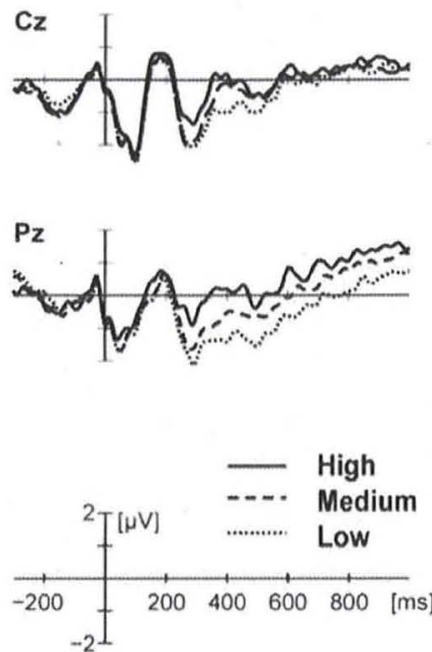

(c)
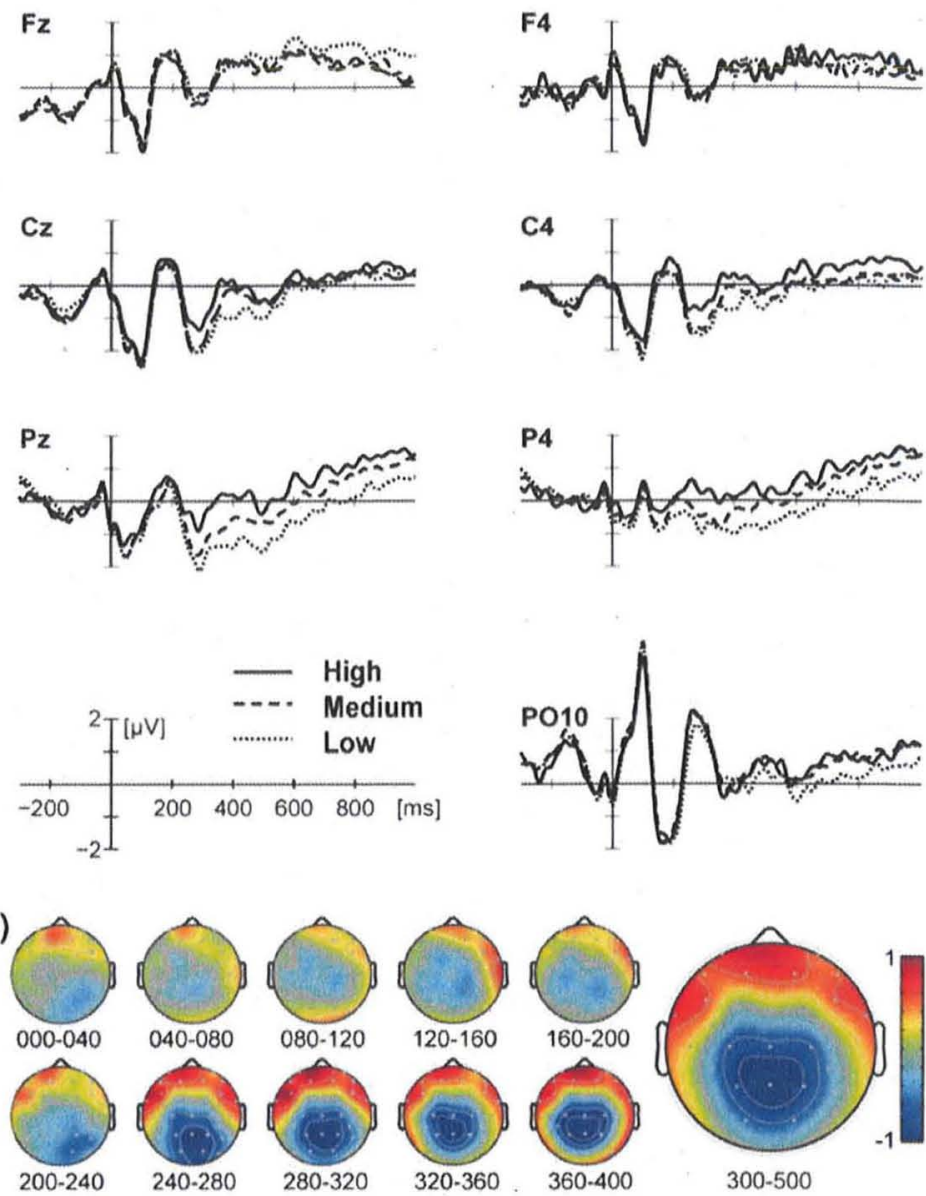

Fig. 6 Predictability effect in fixation related potentials (FRPs). a The grand average FRP, time-locked to first fixation on the target word (time 0 ) shows a graded effect of word predictability that is largest at centroparietal electrode Pz. b Mean horizontal component of the eye track. $\mathbf{c}$ Scalp distributions of the predictability effect (low

we were able to compare our results with these reference datasets.

The EEG was corrected for electro-ocular artifacts with the surrogate Multiple Source Eye Correction method (Berg \& Scherg, 1994), which uses a dipole model of brain activity (Scherg \& Picton, 1991) to achieve optimal separation of ocular artifacts and genuine brain activity. Ocular correction proved to be feasible, and because horizontal ocular artifacts in the EEG correlate highly with the eye tracker signal, we could use the eye tracking information as a new criterion to evaluate the success of artifact correction. Artifact correction allowed us to investigate ERPs time-locked to fixation onset (fixation-related potentials, FRPs) across the entire scalp. The FRP during left-to-right reading is dominated by the lambda response, a visually evoked brain potential that peaks around $100 \mathrm{~ms}$ after fixation onset over visual cortex. We showed that the minus high) are shown for successive $40 \mathrm{~ms}$ windows after fixation onset and for the traditional N400 window (300-500 ms). From Dimigen et al. (2011; Fig. 3; Copyright (2011), with permission from American Psychological Association)

amplitude of the incoming saccade has strong effects both on pre-motor potentials and on the size of the following lambda response. For this reason, it is important to consider saccade amplitude as a covariate in FRP studies, even after eye movement artifacts have been corrected for.

Taking these considerations into account, as one key result, we found that the amplitude of the N400 component in the ERP time-locked to fixation onset (fixation-related potential, FRP) was reliably modulated by the predictability (cloze probability) of the currently fixated word in the sentence context (see Fig. 6). Both size and scalp distribution of predictability effects were very similar to those obtained in the SVP reference dataset. In both paradigms, robust N400 predictability effects arose around $250 \mathrm{~ms}$ after fixation onset or word onset, respectively. However, there was some indication that N400 effects begin earlier under natural reading conditions. As the series of effect 
topographies in Fig. 6c shows, weak N400-like negativities were already visible shortly after the first fixation of word $N$, possibly because word $N$ was parafoveally preprocessed during the previous fixations. Nevertheless, by the time the predictability effect reached its peak in FRPs (around $386 \mathrm{~ms}$ ), readers had in most of the cases $(75 \%)$ already moved on to a different word. This result also supports the proposal that word processing usually lags behind the movement of the eyes during natural reading.

The combined dataset also allowed direct comparisons between word predictability effects on EM (fixation duration) and brain activation (N400 amplitude) for the same set of fixations. We modeled the overall amplitude of the N400 (between 300-500 ms after fixation onset) in order to test whether there is a direct relationship between fixation duration and N400 amplitude that is not mediated by the linguistic properties of the fixated word (e.g., predictability). We found no evidence for such a direct relationship between first-fixation duration and N400, but did find evidence in case of gaze duration, a reading time measure that includes refixations on a word. Similarly, when N400 amplitude was plotted across five levels of predictability, this profile closely resembled the respective profile for refixation probability. Possibly, this means that there are moment-to-moment fluctuations in the efficiency of word recognition that influence both $\mathrm{N} 400$ amplitude and the decision whether a word is refixated or not.

The technical problems of hardware synchronization and ocular artifact correction appear to be solvable (see Dimigen et al., 2011, for further details). Obviously, associated effects need to be taken into account before hypotheses can be tested about timelines of lexical access during reading. However, the use of heterogeneous sentence material with quasi-experimental variations of word properties in our experiment did not allow the extent of experimental control that is desirable to pinpoint ERP correlates of early stages of word identification. To overcome this limitation we constructed sentences in which the same high- and low-frequency target words (1 per sentence) were either highly predictable or not predictable given a lead-in context sentence (Potsdam Sentence Corpus 3 [PSC3]; Dambacher et al. 2009). These sentences were used in three separate (Dambacher, Göllner, Nuthmann, Jacobs, \& Kliegl, 2008) as well as in three combined (Dimigen et al. 2008) EEG and eye movement experiments with a total number of 192 participants.

When the linguistic material was displayed in SVP, the N400 to the unpredicted words started around $200 \mathrm{~ms}$, regardless of whether a slow or a more natural reading-like presentation rate was used. When the sentences were presented for natural reading, the ERPs time-locked to fixation on the target word also revealed a robust predictability effect that was again highly similar in amplitude and scalp distribution to the N400 during SVP. Importantly, relative to SVP the onset of the N400 in natural reading was advanced by more than $100 \mathrm{~ms}$, a result that is reminiscent of the findings by Kretzschmar et al. (2009) with semantic anomalies. Moreover, with the boundary paradigm, which denies preview to the upcoming word by masking we could demonstrate that the early onset of the N400 in natural reading is in fact due to the parafoveal information available in natural reading (Dimigen et al. 2008). When the boundary paradigm was applied to the $N+2$ word (that is, allowing preview of $N+1$, but masking $N+2$ ) the early N400 onset was restored. These results suggest that, during natural reading, intact information from the parafovea permits rapid access to word meaning.

\section{Conclusion}

Reading requires the coordination of two complex systems, word recognition and EM control. Whereas most existent research has focused on one of these systems, we address their interplay with coregistration of EEG and EMs. Where do we stand with respect to simultaneous registration of eye movements and brain electric potentials during this task? Despite years of EM and ERP research, the timeline of word recognition and its relation to oculomotor control is still not understood very well. By combining EM and ERP recordings we aim to (a) compare ERPs in traditional SVP designs and natural reading, (b) describe ERP correlates of oculomotor control and post-saccadic visual processing, (c) examine the determinants for lexical access from a model perspective, and (d) study the impact of the word recognition process on both EMs and ERPs.

Our theoretical framework can be loosely characterized as distributed processing and motivates tests of controversial assumptions implemented in competing computational model of eye-movement control during reading. We distinguish between immediacy, lag, and successor effects. As a preliminary summary, the following picture emerges with respect to effects of word frequency and predictability of words from prior sentence context:

1. Immediacy effects Word frequency and context-based predictability affect early lexical processes and oculomotor control, demonstrating the rapid interplay of visual and expectancy-driven information.

2. Lag effects Word recognition is not limited to the period during which a word is inspected but may continue after fixation and interact with the recognition of the next word. In our view, this may be much more the rule rather than the exception during reading. Thus, oculomotor control and word recognition are partly independent systems, where the eyes can leave a word before processing is completed. 
3. Successor effects The timeline of word recognition strongly depends on parafoveal vision: the preview of upcoming words permit rapid access, which may well extend to their meaning. The denial of preview (e.g., as in traditional SVP studies) induces differences in the timelines of word processing and oculomotor control relative to normal reading.

Past ERP research captured many relevant subprocesses of language comprehension. From SVP tasks, we also learn more and more about how these processes are implemented. We suspect that the basic processing architecture will generalize to natural reading, but that it is likely to go hand in hand with a different parameter specification. Some of these changes will be due to differences in timelines implied by the experimental SVP designs. More dramatic changes are likely due to the dynamic interplay of word recognition and oculomotor control.

In the context of reading, we need to move beyond word recognition and integrate so-called higher-level cognitive processes. Reading and comprehension embodies all of cognition. Some of the best indicators of the comprehension processes are regression saccades carried out in the service of reinspecting previously read material. Obviously, the SVP procedure prevents such processes by design; oculomotor control is necessary for large regressions. Natural reading delivers these measures and will allow us to examine questions, relating for example to the resolution of anaphora (Streb, Rösler, \& Hennighausen, 1999; Streb, Hennighausen, \& Rösler, 2004). We expect that co-registration of eye movements and brain electric potentials will open new windows of opportunity. Despite the increase in complexity of measurement, the constraints enforced by distributed, cascaded processing streams, ideally implemented in computational models of eyemovement control and word recognition, may well lead to more transparent theoretical accounts than the less comprehensive approaches of the past.

\section{References}

Berg, P., \& Scherg, M. (1994). A multiple source approach to the correction of eye artifacts. Electroencephalography and Clinical Neurophysiology, 90(3), 229-241.

Carr, T. H., \& Pollatsek, A. (1985). Recognizing printed words: A look at current models. In D. Besner, T. G. Waller \& G. E. MacKinnon (Eds.), Reading research: Advances in theory and practice (Vol. 5, pp. 1-82). San Diego, CA: Academic Press.

Coltheart, M., Rastle, K., Perry, C., Langdon, R., \& Ziegler, J. (2001). DRC: A dual route cascaded model of visual word recognition and reading aloud. Psychological Review, 108(1), 204-256. doi: 10.1037//0033-295x.108.1.204.

Dambacher, M. (2010). Bottom-up and top-down processes in reading: Influences of frequency and predictability on event-related potentials and eye movements. PhD Thesis. Potsdam: Universitätsverlag Potsdam.

Dambacher, M., Göllner, K., Nuthmann, A., Jacobs, A., \& Kliegl, R. (2008). Frequency and predictability effects on event-related potentials and eye-movements. International Journal of Psychology, 43(3-4), 46-47.

Dambacher, M., \& Kliegl, R. (2007). Synchronizing timelines: Relations between fixation durations and $\mathrm{N} 400$ amplitudes during sentence reading. Brain Research, 1155, 147-162. doi: 10.1016/j.brainres.2007.04.027.

Dambacher, M., Kliegl, R., Hofmann, M., \& Jacobs, A. M. (2006). Frequency and predictability effects on event-related potentials during reading. Brain Research, 1084, 89-103. doi:10.1016/ j.brainres.2006.02.010.

Dambacher, M., Rolfs, M., Göllner, K., Kliegl, R., \& Jacobs, A. M. (2009). Event-related potentials reveal rapid verification of predicted visual input. PLoS ONE, 4(3), e5047. doi:10.1371/ journal.pone.0005047.

Dimigen, O., Sommer, W., Dambacher, M., \& Kliegl, R. (2008) Simultaneous recording of eye movements and ERPs indicates early access to word meaning in natural, left-to-right reading. International Journal of Psychology, 43(3-4), 47.

Dimigen, O., Sommer, W., Hohlfeld, A., Jacobs, A. M., \& Kliegl, R. (2011). Co-registration of eye movements and EEG in natural reading: Analyses and review. Journal of Experimental Psychology: General. doi:10.1037/a0023885.

Dimigen, O., Valsecchi, M., Sommer, W., \& Kliegl, R. (2009). Human microsaccade-related visual brain responses. The Journal of Neuroscience, 29(39), 12321-12331. doi:10.1523/jneurosci. 0911-09.2009.

Engbert, R., \& Kliegl, R. (2001). Mathematical models of eye movements in reading: A possible role for autonomous saccades. Biological Cybernetics, 85(2), 77-87.

Engbert, R., \& Kliegl, R. (2011). Parallel graded attention models of reading. In S. P. Liversedge, I. D. Gilchrist \& S. Everling (Eds.), Eye Movements Handbook. Oxford: Oxford University Press.

Engbert, R., Nuthmann, A., Richter, E. M., \& Kliegl, R. (2005). SWIFT: A dynamical model of saccade generation during reading. Psychological Review, 112(4), 777-813. doi:10.1037/ 0033-295x.112.4.777.

Friederici, A. D., \& Weissenborn, J. (2007). Mapping sentence form onto meaning: The syntax-semantic interface. Brain Research, 1146, 50-58. doi:10.1016/j.brainres.2006.08.038.

Gough, P. B. (1972). One second of reading. Visible Language, 6(4), 291-320.

Graf, R., Nagler, M., \& Jacobs, A. M. (2005). Faktorenanalyse von 57 Variablen der visuellen Worterkennung (Factor analysis of 57 variables in visual word recognition). Zeitschrift für Psychologie, 213(4), 205-218. doi:10.1026/0044-3409.213.4.205.

Grainger, J., \& Jacobs, A. M. (1996). Orthographic processing in visual word recognition: A multiple read-out model. Psychological Review, 103(3), 518-565.

Hagoort, P., Baggio, G., \& Willems, R. M. (2009). Semantic unification. In M. S. Gazzaniga (Ed.), The cognitive neurosciences (4th ed., pp. 819-835). Cambridge, MA: The MIT Press.

Hagoort, P., \& Brown, C. M. (2000). ERP effects of listening to speech compared to reading: The P600/SPS to syntactic violations in spoken sentences and rapid serial visual presentation. Neuropsychologia, 38(11), 1531-1549.

Hagoort, P., \& van Berkum, J. (2007). Beyond the sentence given. Philosophical Transactions of the Royal Society B: Biological Sciences, 362(1481), 801-811. doi:10.1098/rstb.2007.2089.

Henderson, J. M., \& Ferreira, F. (1990). Effects of foveal processing difficulty on the perceptual span in reading: Implications for attention and eye movement control. Journal of Experimental Psychology: Learning, Memory, and Cognition, 16(3), 417-429. 
Inhoff, A. W., Radach, R., Starr, M., \& Greenberg, S. (2000) Allocation of visuo-spatial attention and saccade programming during reading. In A. Kennedy, R. Radach, D. Heller, \& J. Pynte (Eds.), Reading as a perceptual process (pp. 221-246). Amsterdam: Elsevier

Jacobs, A. M., \& Grainger, J. (1994). Models of visual word recognition: Sampling the state of the art. Journal of Experimental Psychology: Human Perception and Performance, 20(6), 1311-1334.

Jost, K., Hennighausen, E., \& Rösler, F. (2004). Comparing arithmetic and semantic fact retrieval: Effects of problem size and sentence constraint on event-related brain potentials. Psychophysiology, 41(1), 46-59. doi:10.1111/1469-8986.00119.

Just, M. A., \& Carpenter, P. A. (1980). A theory of reading: From eye fixations to comprehension. Psychological Review, 87(4), 329-354.

Kennedy, A. (2000). Parafoveal processing in word recognition. Quarterly Journal of Experimental Psychology, 53(2), 429-455.

Kennedy, A., \& Pynte, J. (2005). Parafoveal-on-foveal effects in normal reading. Vision Research, 45(2), 153-168.

Kennedy, A., Pynte, J., \& Ducrot, S. (2002). Parafoveal-on-foveal interactions in word recognition. Quarterly Journal of Experimental Psychology, 55(4), 1307-1337. doi:10.1080/027249 80244000071.

King, J. W., \& Kutas, M. (1995). Who did what and when: Using word-level and clause-level ERPs to monitor working-memory usage in reading. Journal of Cognitive Neuroscience, 7(3), 376-395.

Kliegl, R. (2007). Toward a perceptual-span theory of distributed processing in reading: A reply to Rayner, Pollatsek, Drieghe, Slattery, and Reichle (2007). Journal of Experimental Psychology: General, 136(3), 530-537.

Kliegl, R., \& Engbert, R. (2011). Evaluating a computational model of eye-movement control during reading. In J. H. Wolf (ed.), Models, simulations, and the reduction of complexity. BerlinNew York: Verlag der Akademie. DeGruyter.

Kliegl, R., Grabner, E., Rolfs, M., \& Engbert, R. (2004). Length, frequency, and predictability effects of words on eye movements in reading. European Journal of Cognitive Psychology, 16(1-2), 262-284. doi:10.1080/09541440340000213.

Kliegl, R., Nuthmann, A., \& Engbert, R. (2006). Tracking the mind during reading: The influence of past, present, and future words on fixation durations. Journal of Experimental Psychology: General, 135(1), 12-35. doi:10.1037/0096-3445.135.1.12.

Kolers, P. A. (1976). Buswell's discoveries. In R. A. Monty \& F. W. Senders (Eds.), Eye movements and psychological processes. Hillsdale, NJ: Erlbaum.

Kretzschmar, F., Bornkessel-Schlesewsky, I., \& Schlesewsky, M. (2009). Parafoveal versus foveal N400s dissociate spreading activation from contextual fit. Neuroreport, 20(18), 1613-1618. doi:10.1097/WNR.0b013e328332c4f4.

Kutas, M., \& Federmeier, K. D. (2000). Electrophysiology reveals semantic memory use in language comprehension. Trends in Cognitive Sciences, 4(12), 463-470.

Kutas, M., \& Federmeier, K. D. (2011). Thirty years and counting: Finding meaning in the N400 component of the event-related brain potential (ERP). Annual Review of Psychology, 62(1), 621-647.

McConkie, G. W., \& Rayner, K. (1975). The span of the effective stimulus during a fixation in reading. Perception and Psychophysics, 17(6), 578-586.

McConkie, G. W., Underwood, N. R., Zola, D., \& Wolverton, G. S. (1985). Some temporal characteristics of processing during reading. Journal of Experimental Psychology: Human Perception and Performance, 11(2), 168-186.
Morrison, R. E. (1984). Manipulation of stimulus onset delay in reading: Evidence for parallel programming of saccades. Journal of Experimental Psychology: Human Perception and Performance, 10(5), 667-682.

Niedeggen, M., \& Rösler, F. (1999). N400 effects reflect activation spread during retrieval of arithmetic facts. Psychological Science, 10(3), 271-276.

Niedeggen, M., Rösler, F., \& Jost, K. (1999). Processing of incongruous mental calculation problems: Evidence for an arithmetic N400 effect. Psychophysiology, 36(3), 307-324.

Nuthmann, A., Engbert, R., \& Kliegl, R. (2005). Mislocated fixations during reading and the inverted optimal viewing position effect. Vision Research, 45(17), 2201-2217.

Picton, T. W., Van Roon, P., Armilio, M. L., Berg, P., Ille, N., \& Scherg, M. (2000). The correction of ocular artifacts: A topographic perspective. Clinical Neurophysiology, 111, 53-65.

Pollatsek, A., Reichle, E. D., \& Rayner, K. (2006). Tests of the E-Z Reader model: Exploring the interface between cognition and eye-movement control. Cognitive Psychology, 52(1), 1-56.

Rayner, K. (1975). The perceptual span and peripheral cues in reading. Cognitive Psychology, 7(1), 65-81.

Rayner, K. (1998). Eye movements in reading and information processing: 20 years of research. Psychological Bulletin, 124(3), $372-422$.

Rayner, K. (2009). Eye movements and attention in reading, scene perception, and visual search. Quarterly Journal of Experimental Psychology, 62(8), 1457-1506. doi:10.1080/1747021090281 6461.

Rayner, K., \& Duffy, S. A. (1986). Lexical complexity and fixation times in reading: Effects of word frequency, verb complexity, and lexical ambiguity. Memory and Cognition, 14(3), 191-201.

Rayner, K., \& Pollatsek, A. (1987). Eye movements in reading: A tutorial review. In M. Coltheart (Ed.), Attention and performance 12: The psychology of reading (Vol. 12, pp. 327-362). Hillsdale, NJ: Lawrence Erlbaum Associates.

Rayner, K., Pollatsek, A., Drieghe, D., Slattery, T. J., \& Reichle, E. D. (2007). Tracking the mind during reading via eye movements: Comments on Kliegl, Nuthmann, and Engbert (2006). Journal of Experimental Psychology: General, 136(3), 520-529. doi: 10.1037/0096-3445.136.3.520.

Rayner, K., White, S. J., Kambe, G., Miller, B., \& Liversedge, S. P. (2003). On the processing of meaning from parafoveal vision during eye fixations in reading. In J. Hyönä, R. Radach, \& H. Deubel (Eds.), The Mind's Eye: Cognitive and Applied Aspects of Eye Movement Research (pp. 213-234). Amsterdam: Elsevier.

Reichle, E. D., Pollatsek, A., Fisher, D. L., \& Rayner, K. (1998). Toward a model of eye movement control in reading. Psychological Review, 105(1), 125-157.

Reichle, E. D., Rayner, K., \& Pollatsek, A. (2003). The E-Z Reader model of eye-movement control in reading: Comparisons to other models. Behavioral and Brain Sciences, 26(4), 445-526.

Reichle, E. D., Warren, T., \& McConnell, K. (2009). Using E-Z Reader to model the effects of higher level language processing on eye movements during reading. Psychonomic Bulletin \& Review, 16(1), 1-21. doi:10.3758/pbr.16.1.1.

Reilly, R. G., \& Radach, R. (2006). Some empirical tests of an interactive activation model of eye movement control in reading. Cognitive Systems Research, 7(1), 34-55. doi:10.1016/j.cogsys. 2005.07.006

Roehm, D., Bornkessel-Schlesewsky, I., Rösler, F., \& Schlesewsky, M. (2007). To predict or not to predict: Influences of task and strategy on the processing of semantic relations. Journal of Cognitive Neuroscience, 19(8), 1259-1274.

Rösler, F. (2011). Psychophysiologie der Kognition-Eine Einführung in die Kognitive Neurowissenschaft (Psychophysiology of 
cognition-an introduction to cognitive neuroscience). Heidelberg: Springer.

Scherg, M., \& Picton, T. W. (1991). Separation and identification of event-related potential components by brain electric source analysis. Electroencephalography and Clinical Neurophysiology, pp. 24-37.

Sereno, S. C., \& Rayner, K. (2003). Measuring word recognition in reading: Eye movements and event-related potentials. Trends in Cognitive Sciences, 7(11), 489-493. doi:10.1016/j.tics.2003. 09.010.
Sereno, S. C., Rayner, K., \& Posner, M. I. (1998). Establishing a timeline of word recognition: Evidence from eye movements and event-related potentials. Neuroreport, 9(10), 2195-2200.

Streb, J., Hennighausen, E., \& Rösler, F. (2004). Different anaphoric expressions are investigated by event-related brain potentials. Journal of Psycholinguistic Research, 33(3), 175-201.

Streb, J., Rösler, F., \& Hennighausen, E. (1999). Event-related responses to pronoun and proper name anaphors in parallel and nonparallel discourse structures. Brain and Language, 70(2), 273-286. 